\title{
EDITORIAL
}

\section{La evidencia y el proceso de toma de decisiones}

\section{Evidence and decision making process}

The history of Evidence-Based Medicine is briefly reviewed, and also the concept of evidence used in Evidence-Based Medicine and its relation with the decision making process. Evidence-Based Medicine stresses the examination of evidence from research, especially clinical research, because this kind of evidence is obtained using objective methods and can be criticized. This kind of evidence is used as a masterpiece, together with other elements like social, cultural and economical factors to make the best possible decision. Sometimes evidence and the decision making process are confounded, but in the decision process, objective and subjective elements are combined, to maximally reduce the influence of subjective components. This allows to make the most adequate decision for every patient (Rev Méd Chile 2005; 133: 871-3).

(Key Words: Biomedical research; Decision making; Evidence-Based Medicine)

A mediados de la década de 1980, en Canadá, se inició un nuevo tipo de práctica de la Medicina, caracterizada por el uso sistemático de la evidencia científica en el proceso de toma de decisiones clínicas. Este nuevo tipo de práctica tomó fuerza en la década de 1990, a tal punto que muchos de sus cultores no vacilaron en calificarla como un cambio de paradigma en la práctica de la Medicina ${ }^{1}$. En Chile, esta tendencia (o movimiento como la llaman algunos), empezó a tomar fuerza a fines de 1990 y principios de 2000, comenzando a generarse un debate sobre lo que se entiende por evidencia, la pertinencia de que esta "evidencia" ocupe un lugar central en la práctica de la Medicina por sobre la experiencia clínica y otros tipos de conocimiento, y los peligros que podrían estar involucrados en la práctica de la Medicina Basada en la Evidencia (MBE).

La Revista Médica de Chile publica en este número, un interesante artículo de los Drs. Paula Bedregal y Carlos Cornejo ${ }^{2}$, en el que revisan los orígenes históricos de la $\mathrm{MBE}$, el concepto de evidencia, cuáles serían los presupuestos filosófi- cos de lo que ellos llaman el "movimiento" de la $\mathrm{MBE}$, y hacia dónde se dirige la filosofía de la ciencia y la práctica científica.

$\mathrm{Si}$ bien este artículo aporta mucho material para el debate en relación a la MBE, me parece que contiene algún grado de confusión en cuanto a qué entiende el "movimiento" de la MBE por evidencia y qué se entiende por proceso de toma de decisiones, dos conceptos que si bien en la MBE están muy relacionados, son diferentes.

¿Qué entiende la MBE por evidencia? Por "evidencia" pueden entenderse muchas cosas y, en la práctica, existen distintos tipos de evidencia: empírica, práctica, judicial, etc., y desde ese punto de vista los Drs. Bedregal y Cornejo tienen razón cuando afirman que el concepto de "evidencia" es ambiguo y tiene distintos significados para los profesionales y trabajadores de la salud. Sin embargo, desde sus inicios la MBE ha sido definida como: “... el uso consciente, explícito y juicioso de la mejor evidencia actual, para hacer decisiones acerca del cuidado de pacientes indivi- 
duales"3. Esta definición implica 2 cosas: el uso de la mejor evidencia actual, sin pronunciarse sobre qué tipo de evidencia se trata (de hecho en algunos casos la "mejor" evidencia actual es la opinión de expertos), y un proceso de toma de decisiones basado en dicha evidencia.

Pero, naturalmente, a la MBE no le resulta igual de qué clase de evidencia estamos hablando. Uno de los grandes cultores de la MBE, el Dr. Gordon Guyatt, afirma: "En contraste con el paradigma tradicional de la práctica médica, la MBE reconoce que la intuición, la experiencia clínica no sistemática y el razonamiento fisiopatológico son fundamentos insuficientes para el proceso de decisión clínica y enfatiza el examen de la evidencia proveniente de la investigación clínica"1.

También Sackett apunta a este tipo de evidencia al afirmar: "La MBE es la integración de las mejores evidencias de la investigación con la experiencia clínica y los valores del paciente ${ }^{4}$, y a continuación señala: "Por mejores evidencias de la investigación entendemos investigación relevante, a menudo en ciencias médicas básicas, pero sobre todo investigación clínica centrada en el paciente..."4

Por lo tanto, si bien la MBE reconoce la existencia de distintos tipos de "evidencia", enfatiza el uso de evidencia proveniente de la investigación (especialmente investigación clínica), siendo definida la investigación como un proceso sistemático, organizado y objetivo, destinado a responder una pregunta ${ }^{5}$. Es necesario agregar que, de acuerdo a ciertos teóricos de la investigación, el objetivo último de la investigación (incluida la investigación clínica) es inferir desde los resultados de un estudio, una verdad contenida en el universo ${ }^{6}$.

¿Y por qué la MBE enfatiza el análisis de la evidencia proveniente de la investigación? Porque este tipo de evidencia puede ser sometida a crítica, y este proceso conocido como "Análisis Crítico de la Evidencia Biomédica" es un aspecto central de la MBE. El análisis crítico de la evidencia busca determinar tres cosas: validez (cercanía con la verdad del universo), cuáles son los resultados obtenidos, y aplicación de esos resultados a los pacientes que yo veo (es decir, su utilidad). La validez se determina principalmente por la metodología con que se ha llevado a cabo el estudio, principalmente por la capacidad de los diseños de investigación de controlar los sesgos (aquello que nos aparta sistemáticamente de la verdad) y en este aspecto establece una jerarquía en relación a la capacidad de controlar los sesgos que tienen los distintos diseños de investigación. La aplicación de los resultados se establece por cuán parecidos son los pacientes que yo veo a los pacientes incluidos en el estudio.

Es por tanto la evidencia proveniente de la investigación, especialmente la investigación clínica, publicada y posible de ser sometida a crítica que la MBE enfatiza como aquella que debe ser colocada en el centro del proceso de toma de decisiones, proceso que es en esencia, a diferencia de la evidencia proveniente de la investigación que es objetiva, subjetivo y que tiene siempre un elemento político, ya que, debe tomar en cuenta distintos elementos e integrarlos en la decisión.

Se puede tomar decisiones sin tener en cuenta ninguna evidencia $\mathrm{y}$, de hecho, en la práctica, buena parte de las decisiones se toman de esta manera. Sin embargo, dichas decisiones suelen ser erróneas, ineficientes o inadecuadas. Lo que propone la MBE es colocar la evidencia proveniente de la investigación como un elemento central del proceso de toma de decisiones, a fin de disminuir su subjetividad, pero sin dejar de lado otros elementos importantes y necesarios en este proceso, como son los factores sociales, culturales, de experiencia profesional, restricción de recursos, etc.

De hecho, la definición de MBE propuesta por Sackett y consignada más arriba, explícitamente indica que la MBE es la integración de las mejores evidencias de la investigación con la experiencia clínica y los valores del paciente ${ }^{4}$. Es en el proceso de toma de decisiones y no en la evidencia donde se integran los elementos objetivos y subjetivos que finalmente conducen a una acción. Y cuando una decisión ha sido tomada de esta manera, los resultados son más adecuados que si sólo se consideran elementos subjetivos como la experiencia clínica, por ejemplo. Y si bien de esto no existen evidencias provenientes de experimentos clínicos, sí existen resultados de investigación poblacional que han demostrado que los pacientes que reciben terapias basadas en la evidencia presentan una mejor evolución que los que no la reciben, como es el caso del uso de aspirina y beta bloqueadores en el infarto agudo del miocardio que reduce la mortalidad en comparación a quienes no los usan, la disminución de más de 
$20 \%$ en la mortalidad de los accidentes vasculares encefálicos (AVE) cuando los médicos usan warfarina dosificada de acuerdo a unidades de referencia, y la probabilidad tres veces mayor de sufrir un AVE grave o muerte al mes siguiente en los pacientes sometidos a cirugía de carótida que no siguen los criterios quirúrgicos basados en la evidencia 4 .

Me parece que el artículo de los Drs. Bedregal y Castro aporta elementos muy interesantes y valiosos al debate del valor de la evidencia y su lugar en la práctica de la Medicina, pero tiende a confundir la evidencia con el proceso de toma de decisiones. Evidencias existen muchas y el concepto de evidencia es amplio y puede ser mucho más amplio y diverso en el futuro, pero lo que propone la $\mathrm{MBE}$ es considerar preferentemente la

\section{REFERENCIAS}

1. Guyatt G, Rennie D editores. Users' Guides to the Medical Literature. A manual for Evidence-Based Clinical Practice. AMA Press, Chicago, 2002.

2. Bedregal P, Cornejo C. El movimiento de la Medicina Basada en la Evidencia: alcances conceptuales y teónicos. Rev Méd Chile 2005; 133: 977-82.

3. Sackett D, Rosemberg WM, Gray Ja, Haynes RB, RICHARDSON WS. Evidence based medicine: what it is and what it isn't. BMJ 1996; 312: 71-2. evidencia proveniente de la investigación como el elemento central del proceso de toma de decisiones en Medicina, integrando, además, en este proceso todos los otros elementos necesarios para tomar una decisión (culturales, sociales, valóricos), así como las diversas disciplinas y enfoques que se requieran para que la decisión que se tome sea la más adecuada a las condiciones y necesidades de los pacientes.

\section{Dr. Leandro Biagini A.}

Departamento de Medicina y Centro de Epidemiología Clínica

Facultad de Medicina y Hospital Clínico, Universidad de Chile

Santiago de Chile
4. Sackett D, Strauss SE, Ruchardson WS, Rosenberg W, Haynes RB. Medicina Basada en la Evidencia. Cómo practicar y enseñar la MBE. Segunda edición (español), Hartcourt, 2001.

5. Argimon JMa , JimÉnez J. Métodos de investigación clínica y epidemiológica. Tercera edición, Elsevier España, S.A., 2004.

6. Huley SB, Cummings SR, Browner WS, Grady D, HeARST N, Newman TB. Designing Clinical Research. Second edition, Lippincott Williams \& Wilkins, Philadelphia, USA, 2001. 